\title{
On geometric objects, the non-existence of a gravitational stress- energy tensor, and the uniqueness of the Einstein field equation
}

\author{
Erik Curiel ${ }^{\text {a, b, *, } 1}$ \\ a Munich Center for Mathematical Philosophy, Ludwigstraße 31, Ludwig-Maximilians-Universität, 80539, München, Germany \\ ${ }^{\mathrm{b}}$ Black Hole Initiative, 20 Garden St., Harvard University, Cambridge, MA, 02138, USA
}

\section{A R T I C L E I N F O}

\section{Article history:}

Received 17 August 2018

Accepted 27 August 2018

Available online 8 January 2019

\section{Keywords}

General relativity

Gravitational energy

Stress-energy tensors

Concomitants

Jet bundles

Principle of equivalence

Geometric objects

Einstein field equation

\begin{abstract}
A B S T R A C T
The question of the existence of gravitational stress-energy in general relativity has exercised investigators in the field since the inception of the theory. Folklore has it that no adequate definition of a localized gravitational stress-energetic quantity can be given. Most arguments to that effect invoke one version or another of the Principle of Equivalence. I argue that not only are such arguments of necessity vague and hand-waving but, worse, are beside the point and do not address the heart of the issue. Based on a novel analysis of what it may mean for one tensor to depend in the proper way on another, which, en passant, provides a precise characterization of the idea of a "geometric object", I prove that, under certain natural conditions, there can be no tensor whose interpretation could be that it represents gravitational stress-energy in general relativity. It follows that gravitational energy, such as it is in general relativity, is necessarily non-local. Along the way, I prove a result of some interest in own right about the structure of the associated jet bundles of the bundle of Lorentz metrics over spacetime. I conclude by showing that my results also imply that, under a few natural conditions, the Einstein field equation is the unique equation relating gravitational phenomena to spatiotemporal structure, and discuss how this relates to the non-localizability of gravitational stress-energy. The main theorem proven underlying all the arguments is considerably stronger than the standard result in the literature used for the same purposes (Lovelock's theorem of 1972): it holds in all dimensions (not only in four); it does not require an assumption about the differential order of the desired concomitant of the metric; and it has a more natural physical interpretation.
\end{abstract}

() 2018 Elsevier Ltd. All rights reserved.
As soon as the principle of conservation of energy was grasped, the physicist practically made it his definition of energy, so that energy was that something which obeyed the law of conservation. He followed the practice of the pure mathematician, defining energy by the properties he wished it to have, instead of describing how he measured it. This procedure has turned out to be rather unlucky in the light of the new developments.

Arthur Eddington The Mathematical Theory of Relativity, p. 136

\section{Gravitational energy in general relativity}

There seems to be in general relativity no satisfactory, localized representation of a quantity whose natural interpretation would be "gravitational (stress-)energy". The only physically unquestionable expressions of energetic quantities associated solely with the "gravitational field" we know of in general relativity are quantities derived by integration over non-trivial volumes in spacetimes satisfying any of a number of special conditions. ${ }^{2}$ These quantities,

\footnotetext{
* Munich Center for Mathematical Philosophy, Ludwigstraße 31, Ludwig-Maximilians-Universität, 80539, München, Deutschland, Germany.

E-mail address: erik.curiel@lrz.uni-muenchen.de.

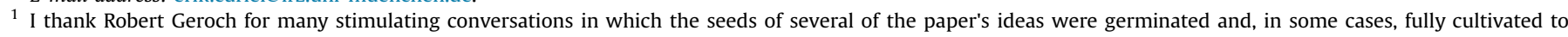

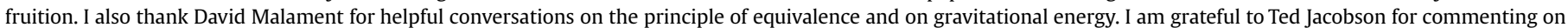
an earlier draft and catching a serious error.

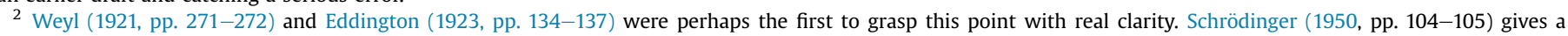

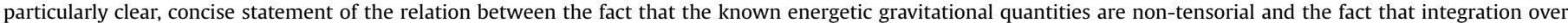
them can be expected to yield integral conservation laws only under restricted conditions.
} 
moreover, tend to be non-tensorial in character. In other words, these are strictly non-local quantities, in the precise sense that they are not represented by invariant geometric objects defined at individual spacetime points (such as tensors or scalars).

This puzzle about the character and status of gravitational energy emerged simultaneously with the discovery of the theory itself. $^{3}$ The problems raised by the seeming non-localizability of gravitational energy had a profound, immediate effect on subsequent research. It was, for instance, directly responsible for Hilbert's request to Noether that she investigate conservation laws in a quite general setting, the work that led to her famous results relating symmetries and conservation laws (Brading, 2005).

Almost all discussions of gravitational energy in general relativity, however, dating back even to the earliest ones, have been plagued by vagueness and lack of precision. The main result of this paper addresses the issue head-on in a precise and rigorous way. Based on an analysis of what it may mean for one tensor to depend in the proper way on another, I prove that, under certain natural conditions, there can be no tensor whose interpretation could be that it represents gravitational stress-energy in general relativity. It follows that gravitational stress-energy, such as it is in general relativity, is necessarily non-local. Along the way, I prove a result of some interest in its own right about the structure of the associated first two jet bundles of the bundle of Lorentz metrics over spacetime. I conclude with a discussion of the sense in which my results also show that the Einstein field equation is, in a natural sense, the unique field equation in the context of a theory such as general relativity, and discuss how this fact relates to the non-localizability of gravitational stress-energy.

The main theorem (7.1) underlying all the arguments is considerably stronger than the standard result in the literature used to argue for the uniqueness of the Einstein field equation (the classic theorem of Lovelock, 1972, stated in footnote 36): it holds in all dimensions, not only in four; it does not require an assumption about the differential order of the desired concomitant of the metric; and it has a more natural physical interpretation. The theorem also has interesting consequences for a proper understanding of the cosmological-constant term in the Einstein field equation, and for higher-dimensional Lanczos-Lovelock theories of gravity, which I discuss at the end of the paper.

\section{The principle of equivalence: $A$ bad argument}

The most popular heuristic argument used to attempt to show that gravitational energy either does not exist at all or does exist but cannot be localized invokes the "principle of equivalence". Choquet-Bruhat (1983, p. 399), for example, puts the argument like this:

This 'non local' character of gravitational energy is in fact obvious from a formulation of the equivalence principle which says that the gravitational field appears as non existent to one observer in free fall. It is, mathematically, a consequence of the fact that the pseudo-riemannian connexion which represents the gravitational field can always be made to vanish along a given curve by a change of coordinates.

\footnotetext{
${ }^{3}$ The first pseudo-tensorial entity proposed to represent gravitational stressenergy dates back to Einstein (1915), the paper in which he first proposed the final form of the theory.
}

Trautman (1976, pp. 135-6) and Goldberg (1980, pp. 469-70) also made essentially the same argument. ${ }^{4}$ Indeed, the making of this argument seems to be something of a shared mannerism among physicists who discuss gravitational energy in general relativity; it is difficult to find an article on the topic in which it is not at least alluded to. ${ }^{5}$

The argument has a fundamental flaw. It assumes that, if there is such a thing as localized gravitational energy or stressenergy, it can depend only on "first derivatives of the metric"-that those first derivatives encode all information about the "gravitational field" relevant to stress-energy - for it is only entities depending only on those first derivatives that one can make vanish along curves. But that seems wrong on the face of it. If there is such a thing as a localized gravitational energetic quantity, then surely it depends on the curvature of spacetime and not on the affine connection (or, more precisely, it depends on the affine connection at least in so far as it depends on the curvature), for any energy one can envision transferring from the gravitational field to another type of system in a different form in general relativity (e.g., as heat or a spray of fundamental particles) must at bottom be based on geodesic deviation, ${ }^{6}$ and so must be determined by the value of the Riemann tensor at a point, not by the value of the affine connection at a point or even along a curve. There is no solution to the Einstein field-equation that corresponds in any natural way to the intuitive Newtonian idea of a constant non-zero gravitational field, i.e., one without geodesic deviation; that, however, would be the only sort of field that one could envision even being tempted to ascribe gravitational energy to in the absence of geodesic deviation, and that attribution is problematic even in Newtonian theory. Indeed, a spacetime has no geodesic deviation if and only if it is everywhere locally isometric to Minkowski spacetime, which we surely want to say has vanishing gravitational energy if any spacetime does, if one can make such a statement precise in the first place. ${ }^{7}$

An obvious criticism of my response to the standard line, related to a popular refinement of the argument given for the nonexistence or non-locality of gravitational energetic quantities, is that it would make gravitational stress-energy depend on secondorder partial derivatives of the field potential (the metric, so comprehended by analogy with the potential in Newtonian theory), whereas all other known forms of stress-energy depend only on terms quadratic in the first partial derivatives of the field potential. To be more precise, the argument runs like this:

\footnotetext{
${ }^{4}$ Goldberg"s formulation of the argument makes explicit a feature at least implicitly common in the many instances I have found in the literature, the conclusion that a local gravitational energy scalar density does not exist and not that a gravitational stress-energy tensor does not exist. One cannot have a scalar energy density for a physical field in general relativity, however, without an associated stress-energy tensor. Such a state of affairs would violate the thermodynamic principle that all energy is equivalent in character, in the sense that any one form can always in principle be tranformed into any other form, since all other known forms of physical field do have a stress-energy tensor as the fundamental representation of their energetic content. I discuss this in more detail in $\S 6$, especially footnote 23 .

5 Bondi (1962), Penrose (1966) and Geroch (1973) are notable exceptions. I take their discussions as models of how one should discuss energetic phenomena in the presence of gravitational fields.

${ }^{6}$ Penrose (1966) and Ashtekar and Penrose (1990) rely on the same idea to very fruitful effect.

7 One might be tempted by the stronger claim that Minkowski spacetime ought to be the unique spacetime with vanishing gravitational energy. I do not think that can be right, however. If the existence of gravitational energy is indeed intimately tied with the presence of geodesic deviation (as argued forcefully by Penrose, 1966), then any flat spacetime, such as that of Kasner (1921), also ought to have vanishing gravitational energy.
} 
One can make precise the sense in which Newtonian gravitational theory is the "weak-field" limit of general relativity (Malament, 1986). In this limit, it is clear that the metric field plays roughly the role in general relativity that the scalar potential $\phi$ does in Newtonian theory. In Newtonian theory, bracketing certain technical questions about boundary conditions, there is a more or less well-defined energy density of the gravitational field, proportional to $(\nabla \phi)^{2}$. One might expect, therefore, based on some sort of continuity argument, or just on the strength of the analogy itself, that any local representation of gravitational energy in general relativity ought to be a "quadratic function of the first partials of the metric". The stress-energy tensor of no other field, moreover, is higher than first-order in the partials of the field potential, so surely gravity cannot be different. No invariant quantity at a point can be constructed using only the first partials of the metric, however, so there can be no scalar or tensorial representation of gravitational energy in general relativity.

(No researcher I know makes the argument exactly in this form; it is just the clearest, most concise version I can come up with myself.) As Pauli (1921, p. 178) forcefully argued, however, there can be no physical argument against the possibility that gravitational energy depends on second derivatives of the metric; the argument above certainly provides none. Just because the energy of all other known fields have the same form in no way implies that a localized gravitational energy in general relativity, if there is such a thing, ought to have that form as well. Gravity is too different a field from others for such a bare assertion to carry any weight. As I explain at the end of $\S 6$, moreover, a proper understanding of tensorial concomitants reveals that an expression linear in second partial derivatives is in the event equivalent in the relevant sense to one quadratic in first order partials. This illustrates how misleading the analogy with Newtonian gravity can be.

\section{Geometric fiber bundles, concomitants, and geometric objects}

The introduction of a coordinate system to geometry is an act of violence.

Hermann Weyl Philosophy of Mathematics and Natural Science

I have argued that, if there is an object that deserves to be thought of as the representation of gravitational stress-energy in general relativity, then it ought to depend on the Riemann curvature tensor. Since there is no obvious mathematical sense in which a general mathematical structure can "depend" on a tensor, the first task is to say what exactly this could mean. I will call a mathematical structure on a manifold that depends in the appropriate fashion on another structure on the manifold, or set of others, a concomitant of it (or them).

The reason I am inquiring into the possibility of a concomitant in the first place, when the question is the possible existence of a representation of gravitational stress-energy is a simple one. What is wanted is an expression for gravitational energy that does not

\footnotetext{
${ }^{8}$ In this light, it is interesting to note that gravitational energy pseudo-tensors do tend to be quadratic in the first-order partials of the metric (Einstein, 1915; Landau \& Lifschitz, 1994; Møller, 1972).
}

depend for its formulation on the particulars of the spacetime, just as the expression for the kinetic energy of a particle in classical physics does not depend on the internal constitution of the particle or on the particular interactions it may have with its environment, and just as the stress-energy tensor for a Maxwell field has the same form as a function of the Faraday tensor in every spacetime irrespective of its particulars. ${ }^{9}$ If there is a well-formed expression for gravitational stress-energy, then one should be able in principle to calculate it whenever there are gravitational phenomena, which is to say, in any spacetime whatsoever-it should be a function of some set of geometric objects associated with the curvature in that spacetime, in some appropriately generalized sense of 'function'. This idea is what a concomitant is supposed to capture.

The term 'concomitant' and the general idea of the thing is due to Schouten (1954, p. 15). ${ }^{10}$ The definition Schouten proposed is expressed in terms of coordinates: depending on what sort of concomitant one deals with, the components of the concomitant in a given coordinate system must satisfy various conditions of covariance under certain classes of coordinate transformations, when those transformations are also applied to the components of the objects the concomitant is defined as a "function" of. His work was picked up and generalized by several other mathematicians, such as Aczél (1960), who extended Schouten's work to treat more generalized classes of higher-order differential concomitants. ${ }^{11}$ The definitions provided by this early work is clear, straightforward and easy to grasp in the abstract, but become difficult to work with in particular cases of interest-Schouten's covariance conditions translate into a set of partial differential equations in a particular coordinate system, which even in seemingly straightforward cases turn out to be forbiddingly complicated. This makes it not only unwieldy in practice and inelegant, but, more important, it makes it difficult to discern what of intrinsic physical significance is encoded in the relation of being a concomitant in particular cases of interest. It is almost impossible to determine anything of the general properties of a particular kind of concomitant of a particular (set of) object(s) by looking at those equations. ${ }^{12}$ I suspect that it is because in particular cases the conditions are so complex, difficult and opaque that use is very rarely made of concomitants in arguments about spacetime structure in general relativity. This is a shame, for

\footnotetext{
9 This property of (stress-)energy for other types of physical systems already stands in contradistinction to the properties of all known rigorous expressions for global gravitational energy in general relativity, e.g., the ADM mass and the Bondi energy, which can be defined only in asymptotically flat spacetimes (Wald, 1984), and most such quasi-local expressions, which can be defined only in stationary or axisymmetric ones (Szabados, 2009).

10 The specific idea of proving the uniqueness of a tensor that "depends" on another tensor, and satisfies a few collateral conditions, dates back at least to Weyl (1921, pp. 315-318) and Cartan (1922). In fact, Weyl proved that the only two-index symmetric covariant tensors one can construct at a point in any spacetime, using only algebraic combinations of the components of the metric and its first two partial derivatives in a coordinate system at that point, that are at most linear in the second derivatives of the metric, are linear combinations of the Ricci curvature tensor, the scalar curvature times the metric and the metric itself. In particular, the only such divergence-free tensors one can construct at a point are linear combinations of the Einstein tensor and the metric with constant coefficients.

11 I thank an anonymous referee for drawing my attention to the work of Aczél and others who developed Schouten"s work.

12 For a good example of just how hairy those conditions can be, see du Plessis (1969, p. 350) for a complete set written out explicitly in the case of two covariant-index tensorial second-order differential concomitants of a Lorentz metric.
} 
the idea is, I think, potentially rich, and so calls out for an invariant formulation. ${ }^{13}$

I use the machinery of fiber bundles to characterize the idea of a concomitant in invariant terms. I give a (brief) explicit formulation of the machinery, because the one I rely on is non-standard. (We assume from hereon that all relevant structures, mappings, etc., are smooth; nothing is lost by the assumption and it simplifies exposition-all germane constructions and proofs can easily be generalized to the case of topological spaces and continuous structures.)

Definition 3.1. A fiber bundle $\mathfrak{B}$ is an ordered triplet, $(\mathscr{B}, \mathscr{M}, \pi)$, such that:
FB1. $\mathscr{B}$ is a differential manifold
FB2. $\mathscr{C}$ is a differential manifold
FB3. $\pi: \mathscr{B} \rightarrow \mathscr{M}$ is smooth and onto
FB4.For every $q, p \in \mathscr{M}, \pi^{-1}(q)$ is diffeomorphic to $\pi^{-1}(p)$ (as submanifolds of $\mathscr{B}$ )
FB5. $\mathscr{B}$ has a locally trivial product structure, in the sense that for each $q \in \mathscr{M}$ there is a neighborhood $U \ni q$ and a diffeomorphism $\zeta$ : $\pi^{-1}[U] \rightarrow U \times \pi^{-1}(q)$ such that the action of $\pi$ commutes with the action of $\zeta$ followed by projection on the first factor.

$\mathscr{B}$ is the bundle space, $\mathscr{M}$ the base space, $\pi$ the projection and $\pi^{-1}(q)$ the fiber over $q$. By a convenient, conventional abuse of terminology, I will sometimes call $\mathscr{B}$ itself 'the fiber bundle' (or 'the bundle' for short). A cross-section $\kappa$ is a smooth map from $\mathscr{M}$ into $\mathscr{B}$ such that $\pi(\kappa(q))=q$, for all $q$ in the mapping's domain.

This definition of a fiber bundle is non-standard in so far as no group action on the fibers is fixed from the start; this implies that no correlation between diffeomorphisms of the base space and diffeomorphisms of the bundle space is fixed. ${ }^{14}$ One must fix that explicitly. On the view I advocate, the geometric character of the objects represented by the bundle arises not from the group action directly, but only after the explicit fixation of a correlation between diffeomorphisms on the base space with those on the bundle space-only after, that is, one fixes how a diffeomorphism on the base space induces one on the bundle. For example, depending on how one decides that a diffeomorphism on the base space ought to

\footnotetext{
13 There is a tradition, initiated in the 1970s by Nijenhuis (1972), that attempts a more invariant formulation of a notion similar to Schouten's original one, introducing the idea of "natural bundles" as a setting for the definition and study of structures closely related to what I call here geometrical objects. That work was elaborated and extended by, e.g., Epstein (1975) and Epstein and Thurston (1979), inter alia. That work is similar to the constructions and arguments I give here. I did not know of it when I developed my own work. (Again, I thank the anonymous referee for drawing my attention to it.) There are two novelties I can claim for my definitions and constructions (besides the fact that it is now all presented in a purely invariant way, with no use of coordinates). First is my definition of fiber bundles without reference to an associated group of transformations, and so the consequent development of what I call geometric bundles based on the idea of inductions. Second, the idea of an induction allows for a simple generalization of my definition for concomitants to more general structures than just tensorial-like objects, e.g., projective structures as characterized by an appropriate family of curves; I do not develop that generalization here as it is not needed. Also, to the best of my knowledge, the main result of $\S 5$, theorem 5.2 , is new, and of some interest in its own right, besides the use I put it to in proving theorem 7.1. (There is some contemporary work being done on so-called natural transformations-e.g., Kolár̆, Michor, \& Slovák, 1993, Koláŕ et al., 1993 and Fatibene \& Francaviglia,and Francaviglia 2003, dating back to Palais \& Terng,and Terng 1977-that bears some similarity to all these ideas, but I do not discuss it, first because it is formulated in category theory and so is fundamentally algebraic in nature, whereas I aim for a formulation with clear and intuitive geometric content, and second because my idea of an induction differentiates my work in important ways from it.)

14 See, e.g., Steenrod (1951) for the traditional definition and the way that a fixed group action on the fibers induces a correlation between diffeomorphisms on the bundle space and those on the base space.
}

induce a diffeomorphism on the bundle over it whose fibers consist of 1-dimensional vector spaces, one will ascribe to the objects of the bundle the character either of ordinary scalars or of $n$-forms (where $n$ is the dimension of the base space). The idea is that the diffeomorphisms induced on the bundle space then implicitly define the group action on the fibers appropriate for the required sort of object. ${ }^{15}$

I call an appropriate mapping of diffeomorphisms on the base space to those on the bundle space an induction. (I give a precise definition in a moment.) In this scheme, therefore, the induction comes first conceptually, and the relation between diffeomorphisms on the base space and those they induce on the bundle serves to fix the fibers as spaces of geometric objects, viz., those whose transformative properties are tied directly and intimately to those of the ambient base space. ${ }^{16}$ This way of thinking of fiber bundles is perhaps not well suited to the traditional mathematical task of classifying bundles, but it turns out to be just the thing on which to base a perspicuous and useful definition of concomitant. Although a diffeomorphism on a base space will naturally induce a unique one on certain types of fiber bundles over it, such as tensor bundles, in general it will not. There is not known, for instance, any natural way to single out a map of diffeomorphisms of the base space into those of a bundle over it whose fibers consist of spinorial objects. ${ }^{17}$ Inductions neatly handle such problematic cases.

I turn now to making this intuitive discussion more precise. A diffeomorphism $\phi^{\sharp}$ of a bundle space $\mathscr{B}$ is consistent with $\phi$, a diffeomorphism of the base space $\mathscr{M}$, if, for all $u \in \mathscr{B}$,

$$
\pi\left(\phi^{\sharp}(u)\right)=\phi(\pi(u))
$$

For a general bundle, there will be scads of diffeomorphisms consistent with a given diffeomorphism on the base space. A way is needed to fix a unique $\phi^{\sharp}$ consistent with a $\phi$ so that a few obvious conditions are met. For example, the identity diffeomorphism on $\mathscr{M}$ ought to pick out the identity diffeomorphism on $\mathscr{B}$. More generally, if $\phi$ is a diffeomorphism on $\mathscr{M}$ that is the identity on an open set $O \subset \mathscr{M}$ and differs from the identity outside $O$, it ought to be the case that the mapping picks out a $\phi^{\sharp}$ that is the identity on $\pi^{-1}[O]$. If this holds, we say that $\phi^{\sharp}$ is strongly consistent with $\phi$.

Let $\mathfrak{D}_{\mathscr{M}}$ and $\mathfrak{D}_{\mathscr{B}}$ be, respectively, the groups of diffeomorphisms on $\mathscr{M}$ and $\mathscr{B}$. Define the set

$\mathfrak{D}_{\mathscr{B}}^{\sharp}=\left\{\phi^{\sharp} \in \mathfrak{D}_{\mathscr{B}}: \exists \phi \in \mathfrak{D}_{\mathscr{M}}\right.$ such that $\phi^{\sharp}$ is strongly consistent with $\phi$. It is simple to show that $\mathfrak{D}_{\mathscr{B}}^{\sharp}$ forms a subgroup of $\mathfrak{D}_{\mathscr{B}}$. This suggests

Definition 3.2. An induction is an injective homomorphism $\iota: \mathfrak{D}_{\mathscr{M}} \rightarrow \mathfrak{D}_{\mathscr{B}}^{\sharp}$. $\phi$ will be said to induce $\phi^{\sharp}$ (under $\iota$ ) if $\iota(\phi)=\phi^{\sharp}$. 18

Definition 3.3. A geometric fiber bundle is an ordered quadruplet $(\mathscr{B}, \mathscr{M}, \pi, \iota)$ where

GFB1. $(\mathscr{B}, \mathscr{M}, \pi)$ satisfies FB1-FB5

GFB2. $\iota$ is an induction Geometric fiber bundles are the appropriate spaces to serve as the domains and ranges of concomitant mappings.

\footnotetext{
15 I will not work out here the details of how this comes about, as they are not needed for the arguments of the paper.

16 See Anderson (1962; 1967), Friedman (1983) and Belot (2011) for other approaches to defining geometric or (as they refer to them) absolute objects.

17 See, e.g., Penrose and Rindler (1984).

18 In a more thorough treatment, one would characterize the way that the induction fixes a group action on the fibers, but we do not need to go into that for our purposes.
} 
Most of the fiber bundles one works with in physics are geometric fiber bundles. A tensor bundle $\mathscr{B}$, for example, is a fiber bundle over a manifold $\mathscr{M}$ each of whose fibers is diffeomorphic to the vector space of tensors of a particular index structure over any point of the manifold; a basis for an atlas is provided by the charts on $\mathscr{B}$ naturally induced from those on $\mathscr{M}$ by the representation of tensors on $\mathscr{M}$ as collections of components in $\mathscr{M}$ 's coordinate systems. There is a natural induction in this case fixed by the pull-back action of a diffeomorphism $\phi$ on tensors on $\mathscr{K}$. Spinor bundles provide interesting examples of physically important bundles that have no natural, unique inductions, though there are classes of them.

We are finally in a position to define concomitants. Let $\left(\mathscr{B}_{1}, \mathscr{M}\right.$, $\left.\pi_{1}, \iota_{1}\right)$ and $\left(\mathscr{B}_{2}, \mathscr{M}, \pi_{2}, \iota_{2}\right)$ be two geometric bundles with the same base space. ${ }^{19}$

Definition 3.4. A mapping $\chi: \mathscr{B}_{1} \rightarrow \mathscr{B}_{2}$ is a concomitant if

$\chi\left(\iota_{1}(\phi)\left(u_{1}\right)\right)=\iota_{2}(\phi)\left(\chi\left(u_{1}\right)\right)$

for all $u_{1} \in \mathscr{B}_{1}$ and all $\phi \in \mathfrak{D} \mathscr{M}$.

In intuitive terms, a concomitant is a mapping between bundles that commutes with the action of the induced diffeomorphisms that lend the objects of the bundles their respective geometrical characters, i.e., the structure in virtue of which they are, in a precise sense, geometric objects. It is easy to see that $\chi$ must be fiberpreserving, in the sense that it maps fibers of $\mathscr{B}_{1}$ to fibers of $\mathscr{B}_{2}$. This captures the idea that the dependence of the one type of object on the other is strictly local; the respecting of the actions of diffeomorphisms captures the idea that the mapping encodes an invariant relation. By another convenient abuse of terminology, I will often refer to the range of the concomitant mapping itself as 'the concomitant' of the domain.

\section{Jet bundles, higher-order concomitants, and geometric objects}

Just as with ordinary functions from one Euclidean space to another, it seems plausible that the dependence encoded in a concomitant from one geometric bundle to another may take into account not only the value of the first geometrical structure at a point of the base space, but also "how that value is changing" in a neighborhood of that point, something like a generalized derivative of a geometrical structure on a manifold. The following construction is meant to capture in a precise sense the idea of a generalized derivative in such a way so as to make it easy to generalize the idea of a concomitant to account for it.

Fix a geometric fibre bundle $(\mathscr{B}, \mathscr{M}, \pi, \iota)$, and the space of its sections $\Gamma[\mathscr{B}]$. Two sections $\gamma, \eta: \mathscr{M} \rightarrow \mathscr{B}$ osculate to first-order at $p \in \mathscr{M}$ if $T \gamma$ and $T \eta$ (the differentials of the mappings) agree in their action on $T_{p} \mathscr{M} .^{20}$ (They osculate to zeroth-order at $p$ if they map $p$ to the same point in the domain.) This defines an equivalence relation on $\Gamma[\mathscr{B}]$. A 1 -jet with source $p$ and target $\gamma(p)$, written ' $j_{p}^{1}[\gamma]$ ', is such an equivalence class. It is not difficult to show that the set of all 1jets,

$J^{1} \mathscr{B}:=\underset{p \in \mathscr{M}, \gamma \in \Gamma[\mathscr{B}]}{\cup} j_{p}^{1}[\gamma]$

\footnotetext{
19 One can generalize the definition of concomitants to cover the case of bundles over different base spaces, but we do not need this here.

20 See, e.g., Hirsch (1976, p. 18) for the definition of the differential of a mapping.
}

naturally inherits the structure of a differentiable manifold (Hirsch, 1976). One can naturally fibre $J^{1} \mathscr{B}$ over $\mathscr{M}$. The source projection $\sigma^{1}: J^{1} \mathscr{B} \rightarrow \mathscr{M}$, defined by

$\sigma^{1}\left(j_{p}^{1}[\gamma]\right)=p$

gives $J^{1} \mathscr{B}$ the structure of a bundle space over the base space $\mathscr{M}$, and in this case we write the bundle $\left(J^{1} \mathscr{B}, \mathscr{M}, \sigma^{1}\right)$. A section $\gamma$ of $\mathscr{B}$ naturally gives rise to a section $j^{1}[\gamma]$ of $J^{1} \mathscr{B}$, the first-order prolongation of that section:

$j^{1}[\gamma]: \mathscr{M} \rightarrow \underset{p \in \mathscr{M}}{\cup} j_{p}^{1}[\gamma]$

such that $\sigma_{1}\left(j^{1}[\gamma](p)\right)=p$. (We assume for the sake of simplicity that global cross-sections exist; the modifications required to treat local cross-sections are trivial, albeit tedious.)

The points of $J^{1} \mathscr{B}$ may be thought of as coordinate-free representations of first-order Taylor expansions of sections of $\mathscr{B}$. To see this, consider the example of the trivial bundle $\left(\mathscr{B}, \mathbb{R}^{2}, \pi\right)$ where $\mathscr{B}:=\mathbb{R}^{2} \times \mathbb{R}$ and $\pi$ is projection onto the first factor. Fix global coordinates $\left(x^{1}, x^{2}, v^{1}\right)$ on $\mathscr{B}$, so that the induced (global) coordinates on $J^{1} \mathscr{B}$ are $\left(x^{1}, x^{2}, v^{1}, v_{1}^{1}, v_{2}^{1}\right)$. Then for any 1 -jet $j_{q}^{1}[\gamma]$, define the inhomogenous linear function $\hat{\gamma}: \mathbb{R}^{2} \rightarrow \mathbb{R}$ by

$\widehat{\gamma}(p)=v^{1}(\gamma(p))+v_{1}^{1}\left(j_{q}^{1}[\gamma]\right)\left(p_{1}-q_{1}\right)+v_{2}^{1}\left(j_{q}^{1}[\gamma]\right)\left(p_{2}-q_{2}\right)$

where $\gamma \in j_{q}^{1}[\gamma]$, and $p, q \in \mathbb{R}^{2}$ with respective components $\left(p_{1}, p_{2}\right)$ and $\left(q_{1}, q_{2}\right)$. Clearly $\widehat{\gamma}$ defines a cross-section of $J^{1} \mathscr{B}$ first-order osculant to $\gamma$ at $p$ and so is a member of $j_{q}^{1}[\gamma]$; indeed, it is the unique globally defined, linear inhomogeneous map with this property.

A 2-jet is defined similarly, by iteration, as an equivalence class of sections under the relation of having the same first and second differentials (as mappings) at a point. More precisely, $\gamma, \eta \in \Gamma[\mathscr{B}]$ osculate to second order at $p \in \mathscr{M}$ if they are in the same 1 -jet and if their second-order differentials equal each other, $T(T \gamma)=T(T \eta)$. Again, this defines an equivalence relation on $\Gamma[\mathscr{B}]$. A 2-jet with source $p$ and target $\gamma(p)$, written ' $j_{p}^{1}[\gamma]$ ', is such an equivalence class. The set of all 2-jets,

$J^{2} \mathscr{B}:=\underset{p \in \mathscr{M}, \gamma \in \Gamma[\mathscr{B}]}{\cup} j_{p}^{2}[\gamma]$

also inherits the structure of a differentiable manifold. $J^{2} \mathscr{B}$ is naturally fibered over $\mathscr{M}$ by the source projection $\sigma^{2}: J^{2} \mathscr{B} \rightarrow \mathscr{M}$, defined by

$\sigma^{2}\left(j_{p}^{2}[\gamma]\right)=p$

giving $J^{2} \mathscr{B}$ the structure of a bundle space over the base space $\mathscr{M}$, $\left(J^{2} \mathscr{B}, \mathscr{K}, \sigma^{2}\right)$. Again, a section $\gamma$ of $\mathscr{B}$ gives rise to a section $j^{2}[\gamma]$ of $J^{2} \mathscr{B}$, the second-order prolongation of that section:

$j^{2}[\gamma]: \mathscr{M} \rightarrow \underset{p \in \mathscr{M}}{\cup} j_{p}^{2}[\gamma]$

such that $\sigma_{1}\left(j^{2}[\gamma](p)\right)=p$. Jet bundles of all higher orders are defined in the same way.

There is a natural projection from $J^{2} \mathscr{B}$ to $J^{1} \mathscr{B}$, the truncation $\theta^{2,1}$, characterized by "dropping the second-order terms in the Taylor expansion". More precisely, for $j^{2}[\gamma]$, the truncation is the 
unique $j^{1}[\eta]$ such that $T j^{1}[\eta]=T T \gamma$, which guarantees that $j^{1}[\eta]=$ $j^{1}[\gamma] .{ }^{21}$ In general, one has the natural truncation $\theta^{n, m}: J^{n} \mathscr{B} \rightarrow J^{m} \mathscr{B}$ for all $0<m<n$.

For our purposes, the most important fact about these spaces is that the jet bundles of a geometric bundle are themselves naturally geometric bundles. Fix a geometric bundle $(\mathscr{B}, \mathscr{M}, \pi, \iota)$ and a diffeomorphism $\phi$ on $\mathscr{M}$. Then $\iota[\phi]$ not only defines an action on points of $\mathscr{B}$, but, as a diffeomorphism itself on $\mathscr{B}$, it naturally defines an action on the cross-sections of $\mathscr{B}$ and thus on the 1 -jets. by the natural pull-back of differentials of mappings. It is easy to show that the mapping $\iota^{1}$ so specified from $\mathfrak{D}_{\mathscr{M}}$ to $\mathfrak{D}_{J^{1} \mathscr{B}}^{\sharp}$ is an injective homomorphism and thus itself an induction; therefore, $\left(J^{1} \mathscr{B}, \mathscr{L}, \sigma^{1}, \iota^{1}\right)$ is a geometric fiber bundle. One defines inductions for higher-order jet bundles in the same way.

We can now generalize our definition of concomitants. Let $\left(\mathscr{B}_{1}\right.$, $\left.\mathscr{M}, \pi_{1}, j\right)$ and $\left(\mathscr{B}_{2}, \mathscr{M}, \pi_{2}, j\right)$ be two geometric fiber bundles over the manifold $\mathscr{M}$.

Definition 4.1. An $n^{\text {th }}$-order concomitant ( $n$ a strictly positive integer) from $\mathscr{B}_{1}$ to $\mathscr{B}_{2}$ is a smooth mapping $\chi: J^{n} \mathscr{B}_{1} \rightarrow \mathscr{B}_{2}$ such that

$1\left(\forall u \in J^{n} \mathscr{B}_{1}\right)\left(\forall \phi \in \mathfrak{U}_{\mathscr{M}}\right) \dot{j}(\phi)(\chi(u))=\chi\left(\iota^{n}(\phi)(u)\right)$

2 there is no $(n-1)^{\text {th }}$-order concomitant $\chi^{\prime}: J^{n-1} \mathscr{B}_{1} \rightarrow \mathscr{B}_{2}$ satisfying

$\left(\forall u \in J^{n} \mathscr{B}_{1}\right) \chi(u)=\chi^{\prime}\left(\theta^{n, n-1}(u)\right)$

A zeroth-order concomitant (or just 'concomitant' for short, when no confusion will arise), is defined by Definition 3.4

An important property of concomitants is that, in a limited sense, they are transitive.

Proposition 4.2. If $\chi_{1}: J^{n} \mathscr{B}_{1} \rightarrow \mathscr{B}_{2}$ is an $n^{\text {th }}$-order concomitant and $\chi_{2}: \mathscr{B}_{2} \rightarrow \mathscr{B}_{3}$ is a smooth mapping, where $\mathscr{B}_{1}, \mathscr{B}_{2}$ and $\mathscr{B}_{3}$ are geometric bundles over the same base space, then $\chi_{2} \circ \chi_{1}$ is an $n^{\text {th }}$-order concomitant if and only if $\chi_{2}$ is a zeroth-order concomitant. This follows directly from the fact that inductions are injective homomorphisms and concomitants respect the fibers.

It will be of physical interest in $\S 6$ to consider the way that concomitants interact with multiplication by a scalar field. (Since we consider in this paper only concomitants of linear and affine objects, multiplication of the object by a scalar field is always defined.) Towards that end, let us say that a concomitant is homogeneous of weight $w$ if for any constant scalar field $\xi$

$\chi\left(\iota_{1}(\phi)\left(\xi u_{1}\right)\right)=\xi^{w} \iota_{2}(\phi)\left(\chi\left(u_{1}\right)\right)$

\section{Concomitants of the metric}

As a specific example that will be of use in what follows, consider the geometric fiber bundle $\left(\mathscr{B} \mathrm{g}, \mathscr{M}, \pi_{\mathrm{g}}, \iota \mathrm{g}\right)$, with $\mathscr{M}$ a 4 dimensional, Hausdorff, paracompact, connected, smooth manifold (i.e., a candidate spacetime manifold), the fibers of $\mathscr{B} \mathrm{g}$ diffeomorphic to the space of Lorentz metrics at each point of $\mathscr{M}$, all of the same signature $(+,-,-,-)$, and $\iota_{\mathrm{g}}$ the induction defined by the natural pull-back. Since the set of Lorentz metrics in the tangent

\footnotetext{
21 One might worry that the truncation is not unique, because two 1-jets may "differ only by a constant" and so still give the same 2 -jet, as may happen with ordinary derivatives in calculus. Because there is no privileged derivative operator on $J^{1} \mathscr{B}$, however, there is no well defined notion of two 1 -jets "differing by a constant".
}

plane over a point of a 4-dimensional manifold, all of the same signature, is a 10 -dimensional manifold, ${ }^{22}$ the bundle space $\mathscr{B}$ g is a 14-dimensional manifold. A cross-section of this bundle defines a Lorentz metric field on the manifold.

The following proposition precisely captures the statement one sometimes hears that there is no scalar or tensorial quantity one can construct depending only on the metric and its first-order partial derivatives at a point of a manifold.

Proposition 5.1. There is no first-order concomitant from $\mathscr{B} \mathrm{g}$ to any tensor bundle over $\mathscr{M}$. To prove this, it suffices to remark that, given any spacetime $\left(\mathscr{M}, g_{a b}\right)$ and any two points $p, p^{\prime} \in \mathscr{M}$, there are open neighborhoods $U$ of $p$ and $U^{\prime}$ of $p^{\prime}$ and a diffeomorphism $\phi: \mathscr{M} \rightarrow \mathscr{M}$, such that $\phi(p)=p^{\prime}, \phi^{\sharp}\left(g_{a b}^{\prime}\right)=g_{a b}$ at $p$, and $\phi^{\sharp}\left(\nabla_{a} g_{b c}\right)=$ $\nabla_{a} g_{b c}$ at $p$, where $\nabla_{a}$ is any derivative operator other than the LeviCivita one associated with $g_{a b}$, and $\phi^{\sharp}$ is the map naturally induced by the pull-back action of $\phi$.

This is not to say, however, that no information of interest is contained in $J^{1} \mathscr{B} \mathrm{g}$. Indeed, two metrics $g_{a b}$ and $h_{a b}$ are first-order osculant at a point if and only if they have the same associated covariant derivative operator at that point. To see this, first note that, if they osculate to first order at that point, then $\widehat{\nabla}_{a}\left(g_{b c}-h_{b c}\right)=$ 0 at that point for all derivative operators. Thus, for the derivative operator $\nabla_{a}$ associated with, say, $g_{a b}, \nabla_{a}\left(g_{b c}-h_{b c}\right)=0$, but $\nabla_{a} g_{b c}=$ 0 , so $\nabla_{a} h_{b c}=0$ at that point as well. Similarly, if the two metrics are equal and share the same associated derivative operator $\nabla_{a}$ at a point, then $\widehat{\nabla}_{a}\left(g_{b c}-h_{b c}\right)=0$ at that point for all derivative operators, since their difference will be identically annihilated by $\nabla_{a}$, and $g_{a b}=h_{a b}$ at the point by assumption. Thus they are first-order osculant at that point and so in the same 1-jet. This proves that all and only geometrically relevant information contained in the 1jets of Lorentz metrics on $\mathscr{K}$ is encoded in the fiber bundle over spacetime the values of the fibers of which are ordered pairs consisting of a metric and the metric's associated derivative operator at a spacetime point.

The second jet bundle over $\mathscr{B}$ g has a similarly interesting structure. Clearly, if two metrics are in the same 2-jet, then they have the same Riemann tensor at the point associated with the 2jet, since the result of doubly applying an arbitrary derivative operator (not the Levi-Civita one associated with the metric) to it at the point yields the same tensor. Assume now that two metrics are in the same 1-jet and have the same Riemann tensor at the associated spacetime point. If it follows that they are in the same 2 -jet, then essentially all and only geometrically relevant information contained in the 2-jets of Lorentz metrics on $\mathscr{M}$ is encoded in the fiber bundle over spacetime the points of the fibers of which are ordered triplets consisting of a metric, the metric's associated derivative operator and the metric's Riemann tensor at a spacetime point. To demonstrate this, it suffices to show that if two Levi-Civita connections agree on their respective Riemann tensors at a point, then the two associated derivative operators are in the same 1-jet of the bundle whose base-space is $\mathscr{K}$ and whose fibers consist of the affine spaces of derivative operators at the points of $\mathscr{M}$ (because they will then agree on the result of application of themselves to their difference tensor, and thus will be in the 2-jet of the same metric at that point).

Assume that, at a point $p$ of spacetime, $g_{a b}=\tilde{g}_{a b}, \nabla_{a}=\tilde{\nabla}_{a}$ (the respective derivative operators), and $R_{b c d}^{a}=\tilde{R}_{b c d}^{a}$ (the respective Riemann tensors). Let $C_{b c}^{a}$ be the symmetric difference-tensor

\footnotetext{
22 In fact, it is diffeomorphic to a connected, convex, open subset-an open cone with vertex at the origin-in $\mathrm{R}^{10}$, and has the further structure of a Fréchet
} manifold (Curiel, 2017). 
between $\nabla_{a}$ and $\tilde{\nabla}_{a}$, which is itself 0 at $p$ by assumption. Then by definition $\nabla_{[b} \nabla_{c]} \xi^{a}=R_{b c n}^{a} \xi^{n}$ for any vector $\xi^{a}$, and so at $p$

$$
\begin{aligned}
R_{a b n}^{c} \xi^{n} & =\nabla_{[a} \tilde{\nabla}_{b]} \xi^{c} \\
& =\nabla_{a}\left(\nabla_{b} \xi^{c}+C_{b n}^{c} \xi^{n}\right)-\tilde{\nabla}_{b} \nabla_{a} \xi^{c} \\
& =\nabla_{a} \nabla_{b} \xi^{c}+\nabla_{a}\left(C_{b n}^{c} \xi^{n}\right)-\nabla_{b} \nabla_{a} \xi^{c}-C_{b n}^{c} \nabla_{a} \xi^{n}+C_{b a}^{n} \nabla_{n} \xi^{c}
\end{aligned}
$$

but $\nabla_{b} \nabla_{c} \xi^{a}-\nabla_{c} \nabla_{b} \xi^{a}=2 R_{b c n}^{a} \xi^{n}$ and $C_{b c}^{a}=0$, so expanding the only remaining term gives

$$
\xi^{n} \nabla_{a} C_{b n}^{c}=0
$$

for arbitrary $\xi^{a}$ and thus $\nabla_{a} C_{c d}^{b}=0$ at $p$; by the analogous computation, $\tilde{\nabla}_{a} C_{c d}^{b}=0$ as well. It follows immediately that $\nabla_{a}$ and $\tilde{\nabla}_{a}$ are in the same 1 -jet over $p$ of the affine bundle of derivative operators over $\mathscr{M}$. We have proven

Theorem 5.2. $J^{1} \mathscr{B} \mathrm{g}$ is naturally diffeomorphic to the geometric fiber bundle over $\mathscr{M}$ whose fibers consist of pairs $\left(g_{a b}, \nabla_{a}\right)$, where $g_{a b}$ is the value of a Lorentz metric field at a point of $\mathscr{M}$, and $\nabla_{a}$ is the value of the covariant derivative operator associated with $g_{a b}$ at that point, the induction being defined by the natural pull-back. $J^{2} \mathscr{B}$ g is naturally diffeomorphic to the geometric fiber bundle over $\mathscr{M}$ whose fibers consist of triplets $\left(g_{a b}, \nabla_{a}, R_{a b c}^{d}\right)$, where $g_{a b}$ is the value of a Lorentz metric field at a point of $\mathscr{M}$, and $\nabla_{a}$ and $R_{a b c}^{d}$ are respectively the covariant derivative operator and the Riemann tensor associated with $g_{a b}$ at that point, the induction being defined by the natural pull-back.

It follows immediately that there is a first-order concomitant from $\mathscr{B}$ g to the geometric bundle $\left(\mathscr{B}_{\nabla}, \mathscr{M}, \pi_{\nabla}, \iota_{\nabla}\right)$ of derivative operators, viz., the mapping that takes each Lorentz metric to its associated derivative operator. (This does not contradict proposition 5.1 , as the bundle of derivative operators is an affine not a tensor bundle.) Likewise, there is a second-order concomitant from $\mathscr{B} \mathrm{g}$ to

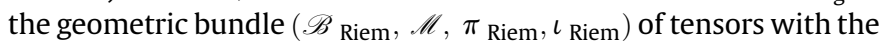
same index structure and symmetries as the Riemann tensor, viz., the mapping that takes each Lorentz metric to its associated Riemann tensor. (This is the precise sense in which the Riemann tensor associated with a given Lorentz metric is "a function of the metric and its partial derivatives up to second order".) It is easy to see, moreover, that both concomitants are homogeneous of degree 0 .

It follows from theorem 5.2 and proposition 4.2 that a concomitant of the metric will be second order if and only if it is a zeroth-order concomitant of the Riemann tensor:

Proposition 5.3. A concomitant of the metric is second-order if and only if it can be expressed as a sum of terms consisting of constants multiplied by the Riemann tensor, the Ricci tensor, the Ricci scalar curvature, and contractions and products of these with the metric itself.

\section{Conditions on a possible gravitational stress-energy tensor}

We are almost in a position to state and prove the main result of the paper, the nonexistence of a gravitational stress-energy tensor. In order to formulate and prove a result having that proposition as its natural interpretation, one must first lay down some natural conditions on the proposed object, to show that no such object exists satisfying the conditions. In general relativity, the stress-energy tensor is the fundamental invariant quantity encoding all known localized energetic properties of all known types of matter field, in the sense that each known type of matter field has a canonical, unique form of stress-energy tensor associated with it, and all other invariant energetic quantities associated with the matter field are derivable from that object. The canonical form of a stress-energy tensor is a two-index, symmetric, covariantly divergence-free tensor. ${ }^{23}$ Not just any such tensor will do, however, for that gives only the baldest of formal characterizations of it. From a physical point of view, at a minimum the object must have the physical dimension of stress-energy for it to count as a stress-energy tensor. That it have the dimension of stress-energy is what allows one to add two of them together in a physically meaningful way to derive the physical sum of total stress-energy from two different sources. In classical mechanics, for instance, both velocity and spatial position have the form of a three-dimensional vector, and so their formal sum is well defined, but it makes no physical sense to add a velocity to a position because the one has dimension length/time and the other the dimension length. (I will give a precise characterization of "physical dimension" below.)

An essential, defining characteristic of energy in classical physics is its obeying some formulation of the First Law of Thermodynamics. The formulation of the First Law I rely on is somewhat unorthodox: that all forms of stress-energy are in principle ultimately fungible-any form of energy can in principle be transformed into any other form ${ }^{24}$-not necessarily that there is some absolute measure of the total energy contained in a system or set of systems that is constant over time. In more precise terms, this means that all forms of energy must be represented by mathematical structures that allow one to define appropriate operations of addition and subtraction among them, which the canonical form of the stress-energy does allow for. ${ }^{25}$ I prefer this formulation of the First Law in general relativity because there will not be in a generic spacetime any well-defined global energetic quantity that one can try to formulate a conservation principle for. In so far as one wants

\footnotetext{
23 Thus, the Bel-Robinson tensor is ruled out from the start, as it is a 4-index tensor. (For characterization and discussion of the Bel-Robinson tensor and its properties, including the way it gives rise to energy-like quantities, see Senovilla, 2000; 2002, Garecki, 2001 and García-Parrado Gómez-Lobo, 2008.) There are indeed several other "energetic quantities" that have in general relativity invariant representation in some form other than a stress-energy tensor, e.g., the ADM mass and various so-called quasi-local quantities (Szabados, 2009). Since none of those are localized quantities, I do not consider them to be relevant to the purposes of this paper. (One might also reasonably complain, so far as my purposes go, that all of those quantities do not differentiate between gravitational and non-gravitational forms of energy, but rather represent only total, aggregate energy.) Starting with Komar (1959) and Finkelstein and Misner (1959), there is another tradition in the context of general relativity of searching for quantities that one might hope to be able to interpret as energetic quantities, possibly associated in a physically relevant way with the "gravitational field", viz., the search for scalar and 1-index objects satisfying various forms of "conservation laws". (See as well, e.g., Trautman, 1962 and Goldberg, 1980.). As interesting as that work is from a mathematical point of view, and as potentially interesting as it may be from a physical point of view, I do not consider here any of those quantities as viable candidates for representations of a localized gravitational energetic quantity, for several reasons. If there are localized energy-like quantities associated with "the gravitational field" in general relativity that do not have the structure of $(0,2)$-index tensor, quantities which are found from investigation of various possible forms of conservation laws, then it seems to me there are two possibilities: there is in fact a gravitational stress-energy tensor, and one can derive those quantities from it, even though that is not how they were discovered; or those quantities are in fact representative of localized gravitational stress-energy, but the claim that they are energetic in some important physical sense has to be articulated and justified, with a particular eye to explaining how such an energy-like quantity interacts with (or not) and is fungible with (or not) the stress-energy content of ordinary matter. I do not know how to do it for any of the objects associated with the search for single-index conservation laws. Indeed, it is striking that none of the researchers who have investigated such objects discuss in any detail the possible physical interpretation of the mathematical structures they were investigating, and in particular how such quantities may relate to what we understand about ordinary stress-energy.

24 Maxwell (1877, ch. v, §97) makes this point especially clearly, including its relation to the principle of energy conservation. See also Maxwell (1888, chs. I, III, IV, VIII, XII).

${ }^{25}$ This kind of linear structure is a requirement even if one takes a more traditional view of the First Law as making a statement about conservation of a magnitude measuring a physical quantity.
} 
to hold on to some principle like the classical First Law in a relativistic context, therefore, I see no other way of doing it besides formulating it in terms of fungibility. (If one likes, one can take the fungibility condition as a necessary criterion for any more traditional conservation law.) This idea is what the demand that all stress-energy tensors, no matter the source, have the same physical dimension is intended to capture. ${ }^{26}$

To sum up, the stress-energy tensor encodes in general relativity all there is to know of ponderable (i.e., non-gravitational) energetic phenomena at a spacetime point:

1 it has 10 components representing with respect to a fixed pseudo-orthonormal frame, say, the 6 components of the classical stress tensor, the 3 components of linear momentum and the scalar energy density of the ponderable field at that point;

2 that it has two covariant indices represents the fact that it defines a linear mapping from timelike vectors at the point ("worldline of an observer") to covectors at that point ("4momentum covector of the field as measured by that observer"), and so defines a bi-linear mapping from pairs of timelike vectors to a scalar density at that point ("scalar energy density of the field as measured by that observer"), because energetic phenomena, crudely speaking, are marked by the fact that they are quadratic in velocity and momental phenomena linear in velocity;

3 that it is symmetric represents, "in the limit of the infinitesimal", the classical principle of the conservation of angular momentum; it also encodes part of the relativistic equivalence of momentum density and the flux of scalar energy density;

4 that it is covariantly divergence-free represents the fact that, "in the limit of the infinitesimal", the classical principles of energy and linear momentum conservation are obeyed; it also encodes part of the relativistic equivalence of momentum-density and scalar energy density flux;

5 the localization of ponderable stress-energy and its invariance as a physical quantity are embodied in the fact that the object representing it is a tensor, a multi-linear map acting only on the tangent and cotangent planes of the point it is associated with ${ }^{27}$;

6 finally, the thermodynamic fungibility of energetic phenomena is represented by the fact that the set of stress-energy tensors forms a vector space-the sum and difference of any two is itself a possible stress-energy tensor, and there is a distinguished zero element-all elements of which have the same physical dimension.

\footnotetext{
${ }^{26}$ For what it's worth, this conception has strong historical warrant-Einstein (implicitly) used a very similar idea in one of his first papers laying out and justifying the general theory (Einstein, 1916, p. 149):

It must be admitted that this introduction of the energy-tensor of matter is not justified by the relativity postulate alone. For this reason we have here deduced it from the requirement that the energy of the gravitational field shall act gravitatively in the same way as any other kind of energy.

Møller (1962) also stresses the fact that the formulation of integral conservation laws in general relativity based on pseudo-tensorial quantities depends crucially on the assumption that gravitational energy, such as it is, shares as many properties as possible with the energy of ponderable (i.e., non-gravitational) matter.
}

27 More generally, the notion of localized quantity I use here means to be represented by a tensor-like object (scalar, tensor, spinor, affine, conformal, projective,

..), one that has values attributable to individual spacetime points and that in some sense or other has properties or actions that ramify into the tangent plane over that point in a way that can be made sense of by restricting attention to the tangent plane.
Consequently, the appropriate mathematical representation of localized gravitational stress-energy, if there is such a thing, is a two covariant-index, symmetric, covariantly divergence-free tensor having the physical dimension of stress-energy. ${ }^{28}$ (That we demand it be covariantly divergence-free is a delicate matter requiring further discussion, which I give at the end of this section.)

Now, in order to make precise the idea of having the physical dimension of stress-energy, recall that in general relativity all the fundamental units one uses to define stress-energy, namely time, length and mass, can themselves be defined using only the unit of time (or equivalently, using only units of length or mass); these are so-called geometrized units (Misner, Thorne, \& Wheeler, 1973, p. 36). ${ }^{29}$ For time, this is trivially true: stipulate, say, that a time-unit is the time it takes a certain kind of atom to vibrate a certain number of times under certain conditions. A unit of length is then defined as that in which light travels in vacuo one time-unit. A unit of mass is defined as that of which two, placed one length-unit apart, will induce in each other by dint of their mutual gravitation alone an acceleration towards each other of one length-unit per time-unit per time-unit. ${ }^{30}$ These definitions of the units of mass and length guarantee that they scale in precisely the same manner as the timeunit when new units of time are chosen by multiplying the timeunit by some fixed real number $\lambda^{-\frac{1}{2}}$. (The reason for the inverse square-root will become clear in a moment). Thus, a duration of $t$ time-units would become $t \lambda^{-\frac{1}{2}}$ of the new units; an interval of $d$ units of length would likewise become $d \lambda^{-\frac{1}{2}}$ in the new units, and $m$ units of mass would become $m \lambda^{-\frac{1}{2}}$ of the new units. This justifies treating all three of these units as "the same", and so expressing acceleration, say, in inverse time-units. To multiply the length of all

\footnotetext{
28 Pitts (2010) has proposed an infinite number of ways to define quantities that he calls representations of localized gravitational energies (all inequivalent). I exclude Pitts"s proposal because I cannot see any physical content to his constructed quantities. How, e.g., could one use one of them to compute the energy a gravitational-wave sensor would absorb from ambient gravitational radiation? Precisely because his quantities depend on the frame one fixes to make the computation, there can be no invariant, physically well defined answer to such a question. If I stick a rod of piezoelectric material in my cup of coffee and use it to warm the coffee from the heat it generates by being deformed by a passing gravitational wave, then surely the rise in temperature of the coffee does not depend on which frame I use to perform the calculation. How should the piezoelectric "know" which of Pitts"s "localized energies" it should draw on? Since there seems to be no way to answer such basic physical questions in an unambiguous way, I do not see that what he has done is to characterize a physical quantity.

29 Aldersley (1977) contains an interesting discussion of geometrized units, and proves a result superficially similar to theorem 7.1, albeit in a very different way than I give here. I have trouble understanding many of his arguments and conclusions, however, as he seems to imply that the physical dimensions of the components of a quantity depend on the physical dimensions of the coordinates in a coordinate system in which the quantity is represented. This makes no sense to me. A quantity simply has a physical dimension, and how one represents it in a coordinate system, if one does at all, is physically irrelevant to that fact.

30 This definition may appear circular, in that it would seem to require a unit of mass in the first place before one could say that bodies were of the same mass. I think the circularity can be mitigated by using two bodies for which there are strong prior grounds for positing that they are of equal mass, e.g., two fundamental particles of the same type. It also suffers from a fundamental lack of rigor that the definition of length does not suffer from. In order to make the definition rigorous, one would have to show that there exists a solution of the Einstein field-equation (approximately) representing two particles in otherwise empty space (as defined by the form of $T_{a b}$ ) - viz., two timelike geodesics-such that, if on a spacelike hypersurface at which they both intersect 1 unit of length apart (as defined on the hypersurface with respect to either) they accelerate towards each other (as defined by relative acceleration of the geodesics) one unit length per unit time squared, then the product of the masses of the particles is 1 . I will just assume, for the purposes of this paper, that such solutions exist. Another possibility for geometrizing a unit of mass would be to define one as that of a Schwarzschild black hole with spatial radius one unit of length, as measured with respect to a fixed radial coordinate respecting the spherical and timelike symmetries of the spacetime. It would be of some interest to determine the relation between these two different ways of
} defining a geometrized unit of mass. 
timelike vectors representing an interval of time by $\lambda^{-\frac{1}{2}}$, however, is equivalent to multiplying the metric by $\lambda$ (and so the inverse metric by $\lambda^{-1}$ ), and indeed such a multiplication is the standard way one represents a change of units in general relativity. This makes physical sense as the way to capture the idea of physical dimension: all physical units, the ones composing the dimension of any physical quantity, are geometrized in general relativity in the most natural formulation, and so depend only on the scale of the metric itself. By Weyl's theorem, however, a metric times a constant represents exactly the same physical phenomena as the original metric (Malament, 2012, ch. 2, $\S 1$ ). ${ }^{31}$

Now, the proper dimension of a stress-energy tensor can be determined by the demand that the Einstein field-equation, $G_{a b}=$ $\gamma T_{a b}$, where $\gamma$ is Newton's gravitational constant, remain satisfied when one rescales the metric by a constant factor. $\gamma$ has dimension $\frac{(\text { length })^{3}}{\left(\text { mass } \text { time }^{2}\right.}$, and so in geometrized units does not change under a constant rescaling of the metric. Thus $T_{a b}$ ought to transform exactly as $G_{a b}$ under a constant rescaling of the metric. A simple calculation shows that $G_{a b}\left(=R_{a b}-1 / 2 R g_{a b}\right)$ remains unchanged under such a rescaling. Thus, a necessary condition for a tensor to represent stress-energy is that it remain unchanged under a constant rescaling of the metric. It follows that the concomitant at issue must be homogeneous of weight 0 in the metric, whatever order it may be.

We must still determine the order of the required concomitant, for it is not a priori obvious. In fact, the weight of a homogeneous concomitant of the metric suffices to fix the differential order of that concomitant. ${ }^{32}$ This can be seen as follows, as exemplified by the case of a two covariant-index, homogeneous concomitant $S_{a b}$ of the metric. A simple calculation based on definition 4.1 and on the fact that the concomitant must be homogeneous shows that the value at a point $p \in \mathscr{M}$ of an $n^{\text {th }}$-order concomitant $S_{a b}$ can be written in the general form

$S_{a b}=\sum_{\alpha} k_{\alpha} g^{q x} \ldots g^{x r}\left(\tilde{\nabla}_{x}^{\left(n_{1}\right)} g_{q x}\right) \ldots\left(\tilde{\nabla}_{x}^{\left(n_{i}\right)} g_{x r}\right)$

where: $\tilde{\nabla}_{a}$ is any derivative operator at $p$ other than the one natu-

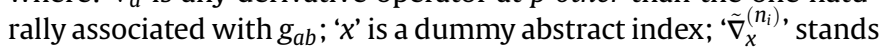
for $n_{i}$ iterations of that derivative operator (obviously each with a different abstract index); $\alpha$ takes its values in the set of all permutations of all sets of positive integers $\left\{n_{1}, \ldots, n_{i}\right\}$ that sum to $n$, so $i$ can range in value from 1 to $n$; the exponents of the derivative operators in each summand themselves take their values from $\alpha$, i.e., they are such that $n_{1}+\cdots+n_{i}=n$ (which makes it an $n^{\text {th }}$-order concomitant); for each $\alpha, k_{\alpha}$ is a constant; and there are just enough of the inverse metrics in each summand to contract all the covariant indices but $a$ and $b$.

Now, a combinatorial calculation shows

Proposition 6.1. If, for $n \geq 2, S_{a b}$ is an $n^{\text {th }}$-order homogeneous concomitant of $g_{a b}$, then to rescale the metric by the constant real number $\lambda$ multiplies $S_{a b}$ by $\lambda^{n-2}$. In other words, the only such homogeneous $n^{\text {th }}$-order concomitants must be of weight $n-2 .{ }^{33}$ So if one knew that $S_{a b}$ were multiplied by, say, $\lambda^{4}$ when the metric was

\footnotetext{
31 Recall that Weyl"s Theorem states that the projective structure and the conformal structure determine the metric up to a constant.

32 I thank Robert Geroch for pointing this out to me.

33 The exponent $n-2$ in this result depends crucially on the fact that $S_{a b}$ has only two indices, both covariant. One can generalize the result for tensor concomitants of the metric of any index structure. A slight variation of the argument, moreover, shows that there does not in general exist a homogeneous concomitant of a given differential order from a tensor of a given index structure to one of another structure-one may not be able to get the number and type of the indices right by contraction and tensor multiplication alone.
}

rescaled by $\lambda$, one would know that it had to be a sixth-order concomitant. In particular, $S_{a b}$ does not rescale when $g_{a b} \rightarrow \lambda g_{a b}$ only if it is a second-order homogeneous concomitant of $g_{a b}$, i.e. (by theorem 5.2 and proposition 5.3), a zeroth-order concomitant of the Riemann tensor. There follows from proposition 4.2

Lemma 6.2. A 2-covariant index concomitant of the Riemann tensor is homogeneous of weight zero if and only if it is a zeroth-order concomitant.

Thus, such a tensor has the physical dimension of stress-energy if and only if it is a zeroth-order concomitant of the Riemann tensor. It is striking how powerful the physically motivated assumption that the required object have the physical dimensions of stressenergy is: it guarantees that the required object will be a secondorder concomitant of the metric.

We now address the issue whether it is appropriate to demand of a potential gravitational stress-energy tensor that it be covariantly divergence-free. In general, I think it is not, even though that is one of the defining characteristics of the stress-energy tensor of ponderable matter in the ordinary formulation of general relativity. ${ }^{34}$ To see this, let $T_{a b}$ represent the aggregate stress-energy of all ponderable matter fields. Let $S_{a b}$ be the gravitational stress-energy tensor, which we assume for the sake of argument to exist. Now, we ask: can the "gravitational field" interact with ponderable matter fields in such a way that stress-energy is exchanged? If it could, then, presumably, there could be interaction states characterized (in part) jointly by these conditions:

$$
\begin{aligned}
& 1 \nabla^{n}\left(T_{n a}+S_{n a}\right)=0 \\
& 2 \nabla^{n} T_{n a} \neq 0 \\
& 3 \nabla^{n} S_{n a} \neq 0
\end{aligned}
$$

It is true that, as ordinarily conceived, condition 2 is incompatible with general relativity as standardly understood and formulated. The existence of a gravitational stress-energy tensor, however, would necessarily entail that we modify our understanding and formulation of general relativity. That is why this argument is only ex hypothesi, and not meant to be one that would make sense in general relativity as we actually know it. (One possible way to understand it, e.g., would be that the ways we currently use to calculate the stress-energy tensor of ordinary matter are mistaken, precisely in so far as they do not take into account possible interactions with gravitational phenomena.)

The most one can say, therefore, without wading into some murkily deep and speculative waters about the way that a gravitational stress-energy tensor (if there were such a thing) might enter into the righthand side of the Einstein field-equation or that its existence might modify the ways we calculate stress-energy for ordinary matter, is that we expect such a thing would have vanishing covariant divergence when the aggregate stress-energy tensor of ponderable matter vanishes, i.e., that gravitational stress-energy on its own, when not interacting with ponderable matter, would be conserved in the sense of being covariantly divergence-free. This weaker statement will suffice for our purposes, so we can safely avoid those murky waters.

Finally, it seems reasonable to require one more condition: were there a gravitational stress-energy tensor, it should not be zero in any spacetime with non-trivial curvature, for one can always envision the construction of a device to extract energy in the presence of curvature by the use of tidal forces and geodesic

\footnotetext{
34 I thank David Malament for helping me get straight on this point. The following argument is in part paraphrastically based on a question he posed to me.
} 
deviation (See, e.g., Bondi and McCrea 1960 and Bondi, 1962, pp. 129-135.)

To sum up:

Condition 6.3. The only viable candidates for a gravitational stressenergy tensor are two covariant-index, symmetric, second-order, zeroweight homogeneous concomitants of the metric that are not zero when the Riemann tensor is not zero and that have vanishing covariant divergence when the stress-energy tensor of ponderable matter vanishes. This discussion, by the way, obviates the criticism of the claim that gravitational stress-energy ought to depend on the curvature, viz., that this would make gravitational stress-energy depend on second-order partial derivatives of the field potential whereas all other known forms of stress-energy depend only on terms quadratic in the first partial derivatives of the field potential. It is exactly second-order, homogeneous concomitants of the metric that possess terms quadratic in the first partials. The rule is that the order of a homogeneous concomitant is the sum of the exponents of the derivative operators when the concomitant is represented in the form of equation (6.1).

\section{Gravitational energy, again, and the Einstein field equation}

If we are to surround ourselves with a perceptual world at all, we must recognize as substance that which has some element of permanence. We may not be able to explain how the mind recognizes as substantial the world-tensor [i.e., the Einstein tensor], but we can see that it could not well recognize anything simpler. There are no doubt minds which have not this predisposition to regard as substantial the things which are permanent; but we shut them up in lunatic asylums.

Arthur Eddington The Mathematical Theory of Relativity, pp. 120-121

It follows from lemma 6.2, in conjunction with condition 6.3, that any candidate gravitational stress-energy tensor must be a zeroth-order concomitant of $\mathscr{B}$ Riem, the geometric bundle of Riemann tensors over spacetime. (One can take this as a precise statement of the fact that any gravitational stress-energy tensor ought to "depend on the curvature", as I argued in §2.) It follows from proposition 5.3 that the only possibilities then are linear combinations of the Ricci tensor and the scalar curvature multiplied by the metric. The only covariantly divergence-free, linear combinations of those two quantities, however, are constant multiples of the Einstein tensor $G_{a b}$. (To see this, note that if there were another, say $k_{1} R_{a b}+k_{2} R_{a b}$ for constants $k_{1}$ and $k_{2}$, then $k_{1} R_{a b}+k_{2} R g_{a b}-2 k_{2} G_{a b}$ would also be divergence free, but that expression is just a constant multiple of the Ricci tensor, which is not in general divergence free.) The Einstein tensor, however, can still be zero even when the Riemann tensor is not (when, e.g., there is only Weyl curvature). This proves the main result.

Theorem 7.1. The only two covariant-index, divergence-free concomitants of the metric that are homogeneous of zero weight are constant multiples of the Einstein tensor. (Note the strength of the result: not only need one not assume that the concomitant be second-order, but one need not even assume the tensor to be symmetric; it all automatically follows from the proof that all such concomitants of the metric are symmetric.) Because the Einstein tensor will be zero in a spacetime having a vanishing Ricci tensor but a non-trivial Weyl tensor, there follows immediately

Corollary 7.2. There are no two covariant-index, divergence-free concomitants of the metric that are homogeneous of weight zero that do not identically vanish when the Riemann tensor is not zero.
The corollary does bear the required natural interpretation, for the Einstein tensor is not an appropriate candidate for the representation of gravitational stress-energy: it can be zero in spacetimes with non-zero curvature; such spacetimes, however, can manifest phenomena, e.g., pure gravitational radiation in the absence of ponderable matter, that one naturally wants to say possess gravitational energy in some (necessarily non-localized) form or other. ${ }^{35}$ Non-localizability does mean that gravitational energy in general relativity, such as it is, is "nowhere in particular", but that is no problem. The same holds for gravitational energy (such as it is) in Newtonian theory, and it also holds for heat in thermodynamics, which is not a localizable quantity, and more generally for work in classical mechanics. That does not mean it is "not in space-time at all", no more than any other globally characterized quantity or entity (e.g., the Euler characteristic of the spacetime manifold, or the incompleteness of an incomplete, inextendible curve, i.e., a singularity, or even the ADM mass) is not. The way such quantities and entities are "in space-time" is a delicate and subtle matter that does call out for investigation and discussion, but this paper is not the place for that. (See Curiel, 2016 for discussion of the question.)

Theorem 7.1 is similar to the classic result of Lovelock (1972), but it is significantly stronger in two important ways. ${ }^{36}$ It does not assume that the desired concomitant be second-order; and it holds in all dimensions, not just four. Both of those properties are grounded on the derivation of the differential order of the desired concomitant of the metric based on analysis of its required physical dimension, encoded in the requirement that the concomitant of the metric be homogeneous of weight zero. The physical interpretation of this is that the desired tensor have the physical dimensions of stress-energy, as is the case for the Einstein tensor, and as must be the case for any tensor that one would equate to a material stressenergy tensor to formulate a field equation (so long as the coupling constant is dimensionless, as is the case for Newton's constant). This provides a physical interpretation to the conditions of the theorem that Lovelock's theorem lacks.

The fact, moreover, that the proof relies essentially only on the structure of the first and second jet bundles of the bundle of metrics over a manifold, i.e., on the bundle of Riemann tensors over a manifold, and how that structure places severe restrictions on its possible concomitants, illuminates the physical and geometrical content of the theorem. Because Lovelock bases his theorem and its proof on Schouten's definition of a concomitant, with the attendant complexity and opacity of the conditions one then has to work with (as I discussed on p. 7, and in particular in footnote 12), his proof consists of several pages of Baroque and unilluminating coordinatebased, brute-force calculation, which gives no physical or

\footnotetext{
35 As an historical aside, it is interesting to note that early in the debate on gravitational energy in general relativity Lorentz (1916) and Levi-Civita (1917) proposed that the Einstein tensor be thought of as the gravitational stress-energy tensor. Einstein criticized the proposal on the grounds that this would result in attributing zero total energy to any closed system.

36 Lovelock proved the following, using the definition of concomitant due to Schouten, and based on earlier work by Rund (1966) and du Plessis (1969).
}

Theorem 7.3. Let $\left(\mathscr{M}, g_{a b}\right)$ be a spacetime. In a coordinate neighborhood of a point $p \in \mathscr{M}$, let $\Theta_{\alpha \beta}$ be the components of a tensor concomitant of $\left\{g_{\lambda \mu} ; g_{\lambda \mu, \nu} ; g_{\lambda \mu, \nu \rho}\right\}$ such that

$\nabla^{n} \Theta_{n b}=0$.
Then

$\Theta_{a b}=r G_{a b}+q g_{a b}$

where $G_{a b}$ is the Einstein tensor and $\mathrm{q}$ and $\mathrm{r}$ are constants. 
geometrical insight into why the theorem holds. The third difference is that the addition of constant multiples of the metric is not allowed. I discuss the consequences of that below.

Before concluding the paper with a discussion of the bearing of the theorem on the Einstein field equation, it behooves us to examine a prima facie puzzle my arguments have left us with. I argued in $\S 6$ that the form of the desired object, that it ought to be a two-index tensor, followed from the idea that all forms of stressenergy ought to be fungible, and so a fortiori one must be able to add in a physically significant way entities representing the stressenergy of different kinds of systems. Now that I have shown that there is no gravitational stress-energy tensor, one may be tempted to conclude that gravitational energy, such as it is, is not fungible with other forms of energy. That would be disastrous, because, as I argued in footnote 28 , there are circumstances whose only reasonable interpretation is that gravitational energy, such as it is, is in some way or other being transformed into other, less recherché forms of energy. (For more rigorous arguments to this effect, again see Bondi and McCrea 1960 and Bondi, 1962, pp. 129-135.) I think the resolution is that, in general relativity, there is no single framework for analyzing and interpreting all the phenomena one may want to characterize as involving the coupling of physical systems based on energy transfer. Energetic concepts that hang together in a unified framework in classical physics come apart in general relativity. When one is dealing with processes mediated by localizable energetic quantities, the stress-energy tensor should do the job; otherwise, there are a multitude of different kinds of quantities any one of which may be physically relevant to the phenomena at issue. This should not be surprising. We already know of cases in which concepts that formed a unified framework in classical physics come apart in radical ways in general relativity, such as the different ways one may characterize a physical system as being in rotation or not (Malament, 2002; 2003). In any event, even in classical physics there are non-localized energetic quantities, such as heat in thermodynamics and gravitational potential energy in Newtonian gravitational theory, that one cannot always treat in a unified framework with all localized forms of energy, and this fact never gave rise to any ambiguities in calculations or other problems.

I conclude the paper by noting that theorem 7.1 has another reasonable interpretation, that, in a natural sense the Einstein field equation is the unique field equation for a theory such as general relativity that unifies spatiotemporal structure with gravitational phenomena by way of an appropriate relation between spacetime curvature and the energetic content of ponderable matter. (In particular, it follows from the result that a cosmological-constant term in the field equation must be construed as forming part of the total stress-energy tensor of spacetime.) Malament (1986) makes precise the sense in which geometrized Newtonian gravity is the limiting theory of general relativity, as "the speed of light goes to infinity". In geometrized Newtonian gravity, moreover, the Poisson equation is formally almost equivalent to the Einstein field equation, and indeed is identical with it in the vacuum case. Malament (2012, ch. 2, §7) argues persuasively that, on this basis, it is natural to adopt the Einstein field equation as the appropriate one when moving from the context of a Newtonian to a relativistic, curved spacetime, in so far as any theory better in some sense than Newtonian theory must, at an absolute minimum, have Newtonian theory as its limit in certain weak-field regimes.

One can read theorem 7.1 as a way to generalize this argument. We know from Newtonian gravitational theory that the intensity of the gravitational field in a spatial region, in so far as one can make sense of this idea, is directly proportional to the density of mass in that region. In geometrized Newtonian gravity, this idea is made precise in the geometrized form of the Poisson equation, which equates a generalized mass-like quantity, which has the form of a stress-energy tensor, to the Ricci curvature of the ambient spacetime. In relativity, one knows that mass just is a form of energy. In order for a relativistic theory of gravitation to have Newtonian gravitational theory as its limiting form, therefore, one is driven to look for the appropriately analogous equation, equating a term representing the curvature of a Lorentz metric with a stress-energy tensor. Once one imposes natural ancillary conditions on the desired curvature term, such as that it must be a second-order, homogeneous concomitant of the metric, then, by theorem 7.1, the Einstein field equation falls out as the only possibility. ${ }^{37}$

Theorem 7.1 implies that the addition of constant multiples of the metric to the geometrical lefthand side of the Einstein field equation is not allowed. I interpret that to mean that any cosmological-constant term must be construed as part of the total stress-energy tensor of spacetime, and so, in particular, the cosmological constant itself must have the physical dimensions of mass $^{2}$, so that its product with the metric will not change under constant rescaling of the metric.

In higher dimensions, there are other tensors satisfying Lovelock's original theorem, the so-called Lovelock tensors. (Those tensors are not linear in the second-order partial-derivatives of the metric as the Einstein tensor is.) Those tensors form the basis of socalled Lanczos-Lovelock gravity theories in dimensions higher than four (Lovelock, 1971; Padmanabhan \& Kothawala, 2013), being used to formulate field equations including Lovelock tensors besides the Einstein tensor. Because theorem 7.1 holds in all dimensions, not just in four, it follows that, in dimensions other than four, the Lovelock tensors are not homogeneous of weight zero, and so do not have the physical dimension of stress-energy. Thus, if one wants to construct a field equation that equates a linear combination of such tensors to the stress-energy tensor of ordinary matter, as Lanczos-Lovelock theories of gravity do, then the coupling constants cannot be dimensionless like Newton's gravitational constant; the physical dimension of each coupling constant will be determined by the physical dimension of the Lovelock tensor it multiplies. These Lovelock tensors are usually interpreted as generalizing the Einstein field equation so as to include curvature terms other than the Einstein tensor that couple with the stressenergy of ponderable matter. As in the case of the cosmological constant, however, the fact that these Lovelock tensors require dimensionful coupling constants to get the physical dimensions of the terms right strongly suggests that one ought not interpret them as geometrical terms coupling to ordinary stress-energy, but rather as exotic forms of stress-energy themselves. If this is correct, then Lanczos-Lovelock theories are not in fact generalizations of general relativity, but rather simply the Einstein field equation with exotic stress-energy added to the righthand side. This is an issue that deserves further investigation.

The fact that the same theorem has as its natural interpretation the uniqueness of the Einstein field equation and the non-existence of a gravitational stress-energy tensor suggests that there may be a tight relation between the non-localizability of gravitational stressenergy and the form of the Einstein field equation. I have a strong suspicion this is correct, but I have not been able to put my finger on exactly what that relation may come to. A hint, perhaps, comes from the pregnant remark of Choquet-Bruhat (1983) to the effect that the principle of equivalence (on her interpretation of it) demands that the gravitational field act as its own source, represented mathematically by the non-linearity of the Einstein field equation.

\footnotetext{
37 One may take this as a more precise and rigorous form of the argument Einstein (1916, p. 149) proposed for his introduction of the stress-energy tensor in the first place, as I discussed in footnote 6 .
} 
Choquet-Bruhat's claim, if true, implies that there can be no linear field equation for gravity satisfying the equivalence principle, which would to my mind be a startlingly strong implication for the equivalence principle to have. And yet my arguments here suggest that she may, in some sense, be correct. That is a question, however, for future work.

I conclude with an intriguing observation. The derivation of the Einstein field equation in Padmanabhan (2010), based on thermodynamical arguments, is really just a special case of theorem 7.1 in disguise, as the Einstein tensor is the only appropriate covariantly divergence-free tensor having the units of stress-energy, as his proof requires. (The same holds true for the generalization of Padmanabhan's arguments to Lanczos-Lovelock gravity in Padmanabhan \& Kothawala, 2013.) Note, moreover, that Lovelock's original theorem does not suffice for Padmanabhan's needs, since it is crucial that the desired tensor have the right physical dimension.

\section{Acknowledgement}

This work was in part funded by a grant from the Deutsche Forschungsgemeinschaft (grant number CU 338/1-1 AOBJ 628412).

\section{References}

Aczél, J. (1959-1960). Ein allgemeines Prinzip bezüglich Komitanten, Differentialkomitanten, kovarianten Ableitungen und Algebren von äquivalenten geometrischen Objekten. Acta Classica Universitas Scientiarum Debreceniensis, 6(2), 5-13.

Aldersley, S. (1977, January). Dimensional analysis in relativistic gravitational theories. Physical Review D, 15(2), 370-376. https://doi.org/10.1103/ PhysRevD.15.370.

Anderson, J. (1962). Absolute change in general relativity. In Recent developments in general relativity (pp. 121-126). Oxford: Pergamon Press. Volume commemorating the 60th birthday of Leopold Infeld, with no editors mentioned by name.

Anderson, J. (1967). Principles of relativity physics. New York: Academic Press.

Ashtekar, A., \& Penrose, R. (1990, October). Mass positivity from focussing and the structure of $i^{\circ}$. Twistor Newsletter, 31, 1-5. Freely available at: http://people maths.ox.ac.uk/lmason/Tn/31/TN31-02.pdf.

Belot, G. (2011, October). Background-independence. General Relativity and Gravitation, 43(10), 2865-2884. https://doi.org/10.1007/s10714-011-1210-x. arXiv: 1106.0920 [gr-qc].

Bondi, H. (1962). On the physical characteristics of gravitational waves. See Lichnerowicz and Tonnelat (1962).

Bondi, H., \& McCrea, W. (1960, October). Energy tranfer by gravitation in Newtonian theory. Mathematical Proceedings of the Cambridge Philosophical Society, 56(4), 410-413. https://doi.org/10.1017/S0305004100034721.

Brading, K. (2005). A note on general relativity, energy conservation, and Noether's theorems. In A. Kox, \& J. Eisenstaedt (Eds.), The Universe of general relativity, number 11 in Einstein studies (pp. 125-135). Boston: Birkhäuser.

Cartan, E. (1922). Sur les Équations de la Gravitation d'Einstein. Journal Mathématiques et Appliqées, 1, 141-203, 9 série.

Choquet-Bruhat, Y. (1983). Two points of view on gravitational energy. In N. Daruelle, \& T. Piran (Eds.), Gravitational radiation (pp. 399-406). Amsterdam: North Holland Press. Proceedings of the NATO Advanced Study Institute, Les Houches Summer School on Gravitational Radiation; Les Houches (France) 02-21 Jun 1982.

Curiel, E. (2016). On the existence of spacetime structure. axw014 The British Journal for the Philosophy of Science. https://doi.org/10.1093/bjps/axw014. A manuscript containing technical appendices working out details of some of the constructions and arguments, and containing further discussion of the possible observability of different kinds of spacetime structure, is available at: arXiv: 1503.03413 [physics.hist-ph] http://strangebeautiful.com/papers/curiel-existst-struct-tech-apdx.pdf.

Curiel, E. (2017). Measure, topology and probabilistic reasoning in cosmology. arXiv: 1509.01878 [gr-qc].

Eddington, A. (1923). Mathematical theory of relativity (2nd ed.). Cambridge: Cambridge University Press.

Einstein, A. (1915). On the general theory of relativity. In The collected papers of Albert Einstein (the Berlin years: Writings, 1914-1917) (Vol. 6, pp. 98-108) Princeton: Princeton University Press. Published originally as "Zur allgemeinen Relativitätstheorie", Sitzungsberichte der Königlich Preußische Akademie der Wissenschaften (Berlin), Gesamtsitzung 4. November 1915:778-785.

Einstein, A. (1916). The foundation of the general theory of relativity. In The principle of relativity (pp. 109-164). New York: Dover Press. https://doi.org/10.1002 andp.19163540702. Published originally as "Die Grundlage der allgemeinen Relativitätstheorie”, Annalen der Physik 49(1916, 7):769-822.
Epstein, D. (1975). Natural tensors on Riemannian manifolds. Journal of Differential Geometry, 10(4), 631-645 (freely downloadable) http://projecteuclid.org/euclid. jdg/1214433166.

Epstein, D., \& Thurston, W. (1979, March). Transformation groups and natural bundles. Proceedings of the London Mathematical society, 38(2), 219-236. https://doi.org/10.1112/plms/s3-38.2.219. s3.

Fatibene, L., \& Francaviglia, M. (2003). Natural and gauge natural formalism for classical field theories: A geometric perspective including spinors and gauge theories. Dordrecht: Kluwer Academic Publishers.

Finkelstein, D., \& Misner, C. (1959, March). Some new conservation laws. Annals of Physics, 6(3), 230-243. https://doi.org/10.1016/0003-4916(59)90080-6.

Friedman, M. (1983). Foundations of space-time theories: Relativistic physics and philosophy of science. Princeton: Princeton University Press.

García-Parrado Gómez-Lobo, A. (2008). Dynamical laws of supergravity in general relativity. Classical and Quantum Gravity, 25(1). https://doi.org/10.1088/02649381/25/1/015006, 015006 arXiv:0707.1475 [gr-qc].

Garecki, J. (2001). Some remarks on the Bel-Robinson tensor. Annalen der Physik, 10(11-12), 911-919. https://doi.org/10.1002/1521-3889(200111)10:11/ $12 \$<\$ 911:: A I D-A N D P 911 \$>\$ 3.0 . C 0 ; 2-M$. arXiv:gr-qc/0003006.

Geroch, R. (1973, December). Energy extraction. Annals of the New York Academy of Sciences, 224, 108-117. https://doi.org/10.1111/j.1749-6632.1973.tb41445.x. Proceedings of the Sixth Texas Symposium on Relativistic Astrophysics.

Goldberg. J. (1980). Invariant transformations, conservation laws, and energy-momentum. In A. Held (Ed.), General relativity and gravitation (Vol. 1, pp. 469-489). New York: Plenum Press, 2 Volumes.

Hirsch, M. (1976). Differential topology. Number 33 in graduate texts in Mathematics. New York: Springer-Verlag.

Kasner, E. (1921). Geometrical theorems on Einstein's cosmological equations. American Journal of Mathematics, 43, 217-221. https://doi.org/10.2307/2370192.

Kolář, I., Michor, P., \& Slovák, J. (1993). Natural operations in differential geometry. Berlin: Springer-Verlag.

Komar, A. (1959). Covariant conservation laws in general relativity. Physical Review, 113, 934-936. https://doi.org/10.1103/PhysRev.113.934.

Landau, L. \& Lifschitz, E. (1994). The classical theory of fields. In Volume 2 of course of theoretical physics (Fourth revised English edition ed.). Amsterdam: Butterworth Heinemann (Translated from the Russian by M. Hammermesh).

Levi-Civita, T. (1917). On the analytic expression that must be given to the gravitational tensor in Einstein's theory. arXiv:physics/9906004v1. A 1999 English translation by S. Antoci and A. Loinger of the original published in Atti della Accademia Nazionale dei Lincei, Rendiconti Lincei. Scienze Fisiche e Naturali, 26(1), 381-391 (Serie 5).

Lichnerowicz, A., \& Tonnelat, A. (Eds.). (1962). Les Théories Relativistes de la Gravitation. Paris: Centre National de la Recherche Scientifique. Number 91 in Colloques Internationaux. Proceedings of a conference held at Royaumont in June, 1959.

Lorentz, H. (1916). Over Einstein's theorie der zwaartekracht (iii). Koninklijke Akademie van Wetenschappen te Amsterdam. In Verslagen van de Gewone vergaderingen der Wisen natuurkundige afdeeling (Vol. 25, pp. 468-486).

Lovelock, D. (1971). The Einstein tensor and its generalizations. Journal of Mathematical Physics, 12(3), 498-501. https://doi.org/10.1063/1.1665613.

Lovelock, D. (1972). The four-dimensionality of space and the Einstein tensor Journal of Mathematical Physics, 13(6), 874-876. https://doi.org/10.1063/ 1.1666069 .

Malament, D. (1986). Newtonian gravity, limits, and the geometry of space. In R. Colodny (Ed.), From quarks to quasars: Philosophical problems of modern physics (pp. 181-201). Pittsburgh: Pittsburgh University Press.

Malament, D. (2002). A no-go theorem about rotation in relativity theory. In D. Malament (Ed.), Reading natural philosophy: Essays in the history and philosophy of science and Mathematics. Chicago: Open Court Press. Essays presented to Howard Stein in honor of his 70th birthday, delivered at a Festchrift in Stein's honor at the University of Chicago, May, 1999.

Malament, D. (2003). On relative orbital rotation in general relativity. In A. Ashtekar (Ed.), Revisiting the foundations of relativistic physics: Festschrift for John Stachel. Dordrecht: Kluwer.

Malament, D. (2012). Topics in the foundations of general relativity and Newtonian gravitational theory. Chicago: University of Chicago Press. Uncorrected final proofs for the book are available for download at http://strangebeautiful.com/ other-texts/malament-founds-gr-ngt.pdf.

Maxwell, J. C. (1877). Matter and motion (Larmor edition). New York: Dover Publications, Inc.. Originally published in 1877. This edition is a 1952 unaltered republication of the 1920 .

Maxwell, J. C. (1888). Theory of heat. Mineola, NY: Dover Publications, Inc. The Dover edition of 2001 republishes in unabridged form the ninth edition of 1888 published by Longmans, Green and Co., London, and also includes the corrections and notes of Lord Rayleigh incorporated into the edition of 1891.

Misner, C., Thorne, K., \& Wheeler, J. (1973). Gravitation. San Francisco: Freeman Press.

Møller, C. (1962). The energy-momentum complex in general relativity and related problems (pp. 15-29). See Lichnerowicz and Tonnelat (1962).

Møller, C. (1972). The theory of relativity (2nd ed.). Oxford: The Clarendon Press. First edition published 1952 .

Nijenhuis, A. (1972). Natural bundles and their general properties. In S. Kobayashi, M. Obata, \& T. Takahashi (Eds.), Differential geometry: In honor of Kentaro yano (pp. 317-334). Tokyo: Kinokuniya Book-store Co. 
Padmanabhan, T. (2010, March). Thermodynamical aspects of gravity: New insights. Reports on Progress in Physics, 73(4). https://doi.org/10.1088/0034-4885/73/4/ 046901, 046901.

Padmanabhan, T., \& Kothawala, D. (2013). Lanczos-Lovelock models of gravity. Physics Reports, 531(3), 115-171. https://doi.org/10.1016/j.physrep.2013.05.007. arXiv:1302.2151 [gr-qc].

Palais, R., \& Terng, C.-L. (1977). Natural bundles have finite order. Topology, 16(3), 271-277. https://doi.org/10.1016/0040-9383(77)90008-8.

Pauli, W. (1921). The theory of relativity. New York: Dover Publications, Inc. A, 1981 reprint of the 1958 edition from Pergamon Press, a translation by G. Field of the original "Relativitätstheorie", in Encyklopädie der matematischen Wissenschaften, vol. V19, B. G. Teubner, Leipzig, 1921.

Penrose, R. (1966). General relativistic energy flux and elementary optics. In B. Hoffman (Ed.), Perspectives in geometry and general relativity (pp. 259-274). Bloomington, IN: Indiana University Press.

Penrose, R., \& Rindler, W. (1984). Spinors and spacetime: Two-spinor calculus and relativistic fields (Vol. 1). Cambridge: Cambridge University Press.

Pitts, J. (2010). Gauge-invariant localization of infinitely many gravitational energies from all possible auxiliary structures. March General Relativity and Gravitation, 42(3), 601-622. https://doi.org/10.1007/s10714-009-0874-y arXiv:0902.1288 [gr-qc].

du Plessis, J. (1969, September). Tensorial concomitants and conservation laws. Tensor, 20, 347-360.

Rund, H. (1966). Variational problems involving combined tensor fields. Abhandlungen aus dem Mathematischen Seminar der Universitat Hamburg, 29(3-4), 243-262. https://doi.org/10.1007/BF03016051. June.

Schouten, J. (1954). Ricci-Calculus: An introduction to tensor analysis and its geometrical applications. In Number $x$ in die grundlehren der mathematischen
Wissenschaften in Einzeldarstellung (2nd ed.). Berlin: Springer-Verlag. First edition published in 1923, in German. The second edition was written in English, and contains significant additions to the first edition.

Schrödinger, E. (1950). Space-time structure. Cambridge science classics. Cambridge: Cambridge University Press. (Reprinted in 1988).

Senovilla, J. (2000). Super-energy tensors. Classical and Quantum Gravity, 17(14) 2799-2842. https://doi.org/10.1088/0264-9381/17/14/313. arXiv:gr-qc/ 9906087.

Senovilla, J. (2002). Superenergy tensors and their applications. Invited lecture pre sented at the 1st Conference on Lorentzian Geometry, "Benalmadena 2001". arXiv: math-ph/0202029.

Steenrod, N. (1951). The topology of fibre bundles. Number 14 in princeton mathematical series. Princeton, NJ: Princeton University Press.

Szabados, L. (2009). Quasi-local energy-momentum and angular momentum in general relativity. Living Reviews in Relativity, 12, 4. https://doi.org/10.12942/lrr2009-4. URL (accessed online 11 Sep 2015) http://www.livingreviews.org/lrr2009-4.Update of lrr-2004-04.

Trautman, A. (1962). Conservation laws in general relativity. In L. Witten (Ed.) Gravitation: An introduction to current research (pp. 169-198). New York: Wiley \& Sons Press.

Trautman, A. (1976). Energy, gravitation and cosmology. In Energy and physics: Proceedings of the 3rd general conference of the european physical society (pp. 133-141). Petit-Lancy, Switzerland: European Physical Society.

Wald, R. (1984). General relativity. Chicago: University of Chicago Press.

Weyl, H. (1921). Space-time-matter (4th ed.). New York: Dover Press. A 1952 reprint of the 1950 translation by H. Brose of the 1921 edition. The first edition published 1918. 